\title{
Robust Attitude Determination for Spacecraft with Magnetic Sensors
}

\author{
By Shoji YoshIKAWA ${ }^{1)}$ and Takehiro NisHIYAMA ${ }^{1)}$ \\ 1) Advanced Technology R\&D Center, Mitsubishi Electric Corporation, Amagasaki, Japan
}

(Received May 2nd, 2008)

\begin{abstract}
This paper presents a new attitude determination algorithm for a spacecraft in low earth orbits using magnetic sensors and rate sensors (e.g. quartz rate sensor gyros) for generic missions including earth observations. The algorithm is applicable to mainly a three-axis spacecraft, including when it is tumbling just after the separation from a launch vehicle or due to some anomaly. The advantage of the proposed approach is that it does not require additional attitude sensors such as sun sensors. First, an attitude determination algorithm using only magnetic sensors is introduced presuming that the attitude change during the measurement period is very small. Then the algorithm is extended to combine magnetic sensors with rate sensors, where both the three-axis attitude and rate sensor biases are estimated. The estimation error is statistically analyzed. Numerical examples are given to validate the proposed algorithm and its accuracy evaluation.
\end{abstract}

Key Words: Attitude Determination, Magnetic Sensors, Rate Sensors

\section{Nomenclature}

\begin{tabular}{|c|c|}
\hline$J$ & : cost function \\
\hline$N$ & : number of measurements \\
\hline$t$ & : time \\
\hline$\Delta t$ & : sampling time \\
\hline$\delta_{i j}$ & : Kronecker's delta \\
\hline$\sigma_{v}$ & $\begin{array}{l}\text { standard deviation of the small angle } \\
\text { rotations }\end{array}$ \\
\hline$\theta$ & $\begin{array}{l}\text { total change in the direction of the } \\
\text { magnetic vector }\end{array}$ \\
\hline$\theta_{i}$ & : rotation angle of $r_{i}$ from $r$ \\
\hline$r$ & : magnetic field vector \\
\hline $\mathbf{v}$ & : small angle rotation \\
\hline$w$ & : measurement error vector \\
\hline$\omega$ & : body rate \\
\hline$I_{3}$ & : $3 \times 3$ identity matrix \\
\hline $\boldsymbol{U}$ & : coordinate transformation matrix \\
\hline \multicolumn{2}{|c|}{ Superscripts } \\
\hline mes & measured value \\
\hline$B$ & expressed in the body coordinates \\
\hline$I$ & expressed in the inertial coordinates \\
\hline$T$ & $\begin{array}{l}\text { transpose of the vector or the matrix in } \\
\text { question }\end{array}$ \\
\hline$\wedge$ & estimated value \\
\hline$\sim$ & estimation error \\
\hline- & unit vector \\
\hline \multicolumn{2}{|c|}{ Subscripts } \\
\hline$b$ & bias in the sensor output \\
\hline$i$ & i-th measurement \\
\hline \multicolumn{2}{|l|}{ Operators } \\
\hline$[\varepsilon \times]$ & $\begin{array}{l}3 \times 3 \text { cross-product-equivalent matrix } \\
\text { associated with the } 3 \text {-dimensional vector } \\
\varepsilon\end{array}$ \\
\hline$E[$ & operation of taking an expectation \\
\hline
\end{tabular}

\section{Introduction}

Attitude determination of spacecraft in low earth orbits using magnetic sensors is attractive. Firstly, they are inexpensive, small and lightweight. Secondly, they are robust and complete in the sense that sun sensors and star sensors are vulnerable to occultation ${ }^{1)}$. Furthermore, magnetic sensors are applicable to wide-range of body rate. They can provide rate information as a backup for gyros ${ }^{2}$.

However, magnetic sensors have a drawback. A single measurement gives a two axis information. To determine a three-axis attitude, additional information is required.

Conventionally, this drawback has been solved by either method a) or b).

a) to use an additional attitude sensor such as a sun sensor or an earth sensor assuming that the attitude is spin-stabilized

b) to take another measurement of the magnetic field assuming that attitude change information during the two measurements is available from magnetic sensor outputs

Method a) is illustrated by Figure 1, where a spinning spacecraft is equipped with a three-axis magnetic sensor and a slit-type sun sensor. Because the spin axis is maintained in the inertial frame, intermittent information from a sun sensor is sufficient to construct a three-axis attitude determination ${ }^{3)}$. Obviously method a) has a drawback that it is not applicable to a three-axis spacecraft and that it adds another attitude sensor and increases cost and weight.

On the other hand, in method b), we do not add attitude sensors. Instead, we wait for a while so that the orbital motion of the spacecraft brings a well different position in the earth magnetic field. Because spacecraft cannot be totally stationary under natural disturbances, the attitude change information during the two measurements is required. Rate estimation using only magnetic sensor outputs is possible ${ }^{4)}$ and estimation of attitude and rate using only magnetic sensors has 
been proposed ${ }^{2), 5)}$. The method $b$ ) has a drawback that deriving rate estimation from magnetic sensor outputs requires time derivative of magnetic sensor outputs, which tends to induce noisy results and needs a sophisticated filtering.

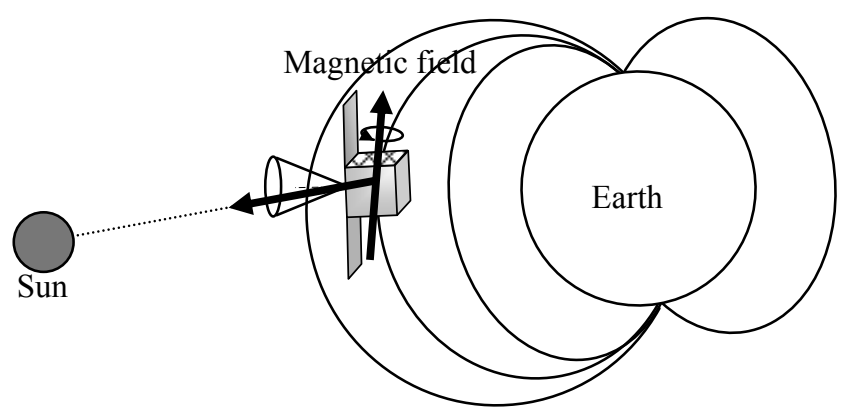

Fig. 1. Conventional attitude sensor configuration

This paper presents a solution to the above mentioned drawbacks of the magnetic sensors and conventional methods.

Considering that rate sensors are lightweight and inexpensive, it is practically reasonable to combine rate sensors with magnetic sensors. It is known that rate sensor biases are not negligible. Therefore, this paper proposes to use rate sensors to estimate the attitude change, whereas the rate sensor biases are simultaneously estimated using magnetic sensor outputs.

This paper is organized as follows. In section 2, an attitude determination algorithm using only magnetic sensors is introduced presuming that the spacecraft attitude is stationary. In section 3, the algorithm is extended to combine magnetic sensors with rate sensors, where both the three-axis attitude and rate sensor biases are estimated. Section 4 gives numerical examples. Section 5 concludes the paper.

\section{Stationary Attitude Determination by Magnetic Sensors}

We discuss an attitude determination algorithm using only magnetic sensors presuming that the attitude change during the measurement period is small enough that the spacecraft attitude can be regarded as stationary.

\subsection{Measurement model}

Three-axis magnetic sensors measure the local geomagnetic field in the body coordinates. After calibration of their scale factors and biases, their outputs have a simple relationship with the magnetic field vector given by a geomagnetic field model such as the International Geomagnetic Field Model (IGRF) as ${ }^{6), 7)}$

$$
\boldsymbol{U}_{B \rightarrow I}\left(\boldsymbol{r}^{B}+\boldsymbol{w}\right)=\boldsymbol{r}^{I}
$$

The direction of the vectors $\boldsymbol{r}^{B}$ and $\boldsymbol{r}^{I}$ is used for attitude estimation and their magnitude is not used. Therefore, $\boldsymbol{r}^{B}$ and $\boldsymbol{r}^{I}$ are regarded as normalized in the following discussion. Also, the bias components in the magnetic sensor outputs are regarded as negligible after the calibration of the scale factors and biases. Furthermore, the IGRF model error is considered negligible compared with magnetic sensor output errors if the latest IGRF model is used under a quiet environment (i.e. not during or just after the magnetic storm). A vector RMS error of IGRF model is evaluated less than 100 $\mathrm{nT}^{8)}$, whereas a typical magnetic sensor output error is on the order of micro $\mathrm{T}$.

One measurement gives two degree-of-freedom (d.o.f.) information. To determine a three-axis attitude, it is necessary to take measurements at least two points that are enough separated from each other (Fig. 2).

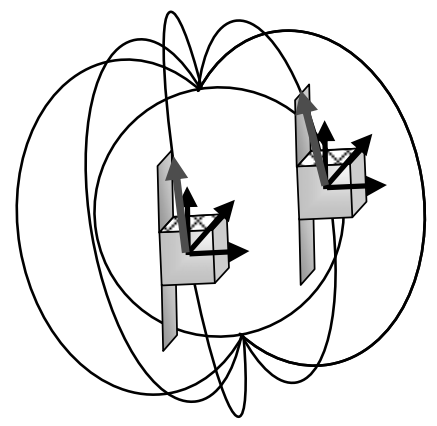

Fig. 2. Measurements at two points (stationary case)

To average out the random errors contained in Eq. (1), we take $N$ measurements. Then the attitude estimation problem is to find a coordinates transformation matrix $\boldsymbol{U}_{B \rightarrow I}$ that satisfies the following relationship.

$$
\boldsymbol{U}_{B \rightarrow I}\left(\boldsymbol{r}_{i}^{B}+\boldsymbol{w}_{i}\right)=\boldsymbol{r}_{i}^{I} \quad(i=1, \cdots, N)
$$

\subsection{Attitude determination algorithm}

Suppose that two measurements by magnetic sensors provide a magnetic unit vector in the body coordinates at each orbit position. Suppose also that earth magnetic field model provides a magnetic unit vector in the earth fixed coordinates (which can be easily converted into the inertial coordinates). Then a simple method (algebraic method) is known that constitutes an orthogonal frame from two unit vectors in each coordinates and computes a coordinates transformation matrix (equivalently a three-axis attitude) by combining the two orthogonal frames ${ }^{6}$. We determine the coordinates transformation matrix $\boldsymbol{U}_{B \rightarrow I}$ using algebraic method.

Because the measurement errors of the magnetic sensors are comparatively large, two measurements may not achieve sufficient accuracy in attitude determination. Therefore, we take $\mathrm{N}$-times measurements and solve for a coordinates transformation matrix that match $N$ vectors from sensors to $N$ vectors from the model. We define a cost function $J$ and find $\boldsymbol{U}_{B \rightarrow I}$ that minimizes $J$.

$$
\min _{\boldsymbol{U}_{B \rightarrow I}} J\left(\boldsymbol{U}_{B \rightarrow I}\right)=\sum_{i=1}^{N}\left\|\boldsymbol{r}_{i}^{I}-\boldsymbol{U}_{B \rightarrow I} \boldsymbol{r}_{i}^{B}\right\|^{2}
$$

We start with the three-axis attitude estimated by the algebraic method; $\hat{\boldsymbol{U}}_{B \rightarrow I}$ and update the attitude estimates based on the differences between the measured vectors and vectors that are transformed near to the measured ones through the initial estimated coordinates transformation matrix.

$$
J(\delta \boldsymbol{U})=\sum_{i=1}^{N}\left\|\hat{\boldsymbol{U}}_{B \rightarrow I}^{T} \boldsymbol{r}_{i}^{I}-\delta \boldsymbol{U} \boldsymbol{r}_{i}^{B}\right\|^{2}
$$




$$
\hat{\boldsymbol{U}}_{B \rightarrow I} \delta \boldsymbol{U} \rightarrow(\text { new }) \hat{\boldsymbol{U}}_{B \rightarrow I}
$$

Noting that the attitude estimate update $\delta \boldsymbol{U}$ is small, we apply small angle approximations to $\delta \boldsymbol{U}$.

$$
\delta \boldsymbol{U} \approx \boldsymbol{I}_{3}-[\varepsilon \times]
$$

By its definition, $\boldsymbol{\varepsilon}$ gives an estimate update in the body coordinates. Substituting Eq. (6) into Eq. (4), we derive linearized equations in terms of attitude estimate update.

$$
J \approx \sum_{i=1}^{N}\left\|\Delta \boldsymbol{r}_{i}^{B}-\boldsymbol{B}_{i} \boldsymbol{\varepsilon}\right\|^{2}
$$

where a residual vector $\Delta \boldsymbol{r}_{i}^{B}$ and a matrix $\boldsymbol{B}_{i}$ are defined as

$$
\Delta \boldsymbol{r}_{i}^{B}=\hat{\boldsymbol{U}}_{B \rightarrow I}^{T} \boldsymbol{r}_{i}^{I}-\boldsymbol{r}_{i}^{B}, \quad \boldsymbol{B}_{i}=\left[\boldsymbol{r}_{i}^{B} \times\right] .
$$

Following the standard least square method, we obtain the optimal attitude update $\hat{\boldsymbol{\varepsilon}}$ as

$$
\hat{\boldsymbol{\varepsilon}}=\left(\sum_{i=1}^{N} \boldsymbol{B}_{i}^{T} \boldsymbol{B}_{i}\right)^{-1} \sum_{i=1}^{N} \boldsymbol{B}_{i}^{T} \Delta \boldsymbol{r}_{i}^{B}
$$

If we compute new $\hat{\boldsymbol{U}}_{B \rightarrow I}$ using Eq. (5), regard it as an initial estimate and iterate the process of Eqs. (8),(9) and (5), we can improve estimation accuracy.

\subsection{Evaluation of estimation accuracy}

The measured magnetic field vector contains a measurement error. Because the magnetic field vector measurement is normalized, this error is modeled as a small angle rotation.

$$
\boldsymbol{w}_{i}=\left[\boldsymbol{v}_{i} \times\right] \boldsymbol{r}_{i}^{B}
$$

The small angle rotation $\boldsymbol{v}_{i}$ is a zero-mean random vector as is mentioned in subsection 2.1 and is supposed to be Gaussian.

$$
E\left[\boldsymbol{v}_{i}\right]=\boldsymbol{0}, \quad E\left[\boldsymbol{v}_{i} \boldsymbol{v}_{j}^{T}\right]=\sigma_{v}^{2} \delta_{i j} \boldsymbol{I}_{3}
$$

From Eqs. (9) and (10), estimation errors $\varepsilon$ are computed as

$$
\tilde{\boldsymbol{\varepsilon}}=\hat{\boldsymbol{\varepsilon}}-\boldsymbol{\varepsilon}=\left(\sum_{i=1}^{N} \boldsymbol{B}_{i}^{T} \boldsymbol{B}_{i}\right)^{-1} \sum_{i=1}^{N} \boldsymbol{B}_{i}^{T}\left[\boldsymbol{v}_{i} \times\right] \boldsymbol{r}_{i}^{B} .
$$

From Eqs. (11) and (12), statistical evaluation of attitude estimation errors is obtained as

$$
E[\tilde{\boldsymbol{\varepsilon}}]=\boldsymbol{0}, \quad E\left[\tilde{\boldsymbol{\varepsilon}} \tilde{\boldsymbol{\varepsilon}}^{T}\right]=\sigma_{v}^{2}\left(\sum_{i=1}^{N} \boldsymbol{B}_{i}^{T} \boldsymbol{B}_{i}\right)^{-1} .
$$

Noting that the following identity holds for an arbitrary $3 \mathrm{x} 1$ unit vector $\boldsymbol{x}$,

$$
[\boldsymbol{x} \times][\boldsymbol{x} \times]^{T}=\boldsymbol{I}_{3}-\boldsymbol{x} \boldsymbol{x}^{T}
$$

we rewrite the second part of Eq. (13) into

$$
E\left[\tilde{\boldsymbol{\varepsilon}} \tilde{\boldsymbol{\varepsilon}}^{T}\right]=\sigma_{v}^{2}\left(\sum_{i=1}^{N} \boldsymbol{I}_{3}-\boldsymbol{r}_{i}^{B} \boldsymbol{r}_{i}^{B^{T}}\right)^{-1} .
$$

This expression is essentially the same as Shuster's ${ }^{9)}$, where he uses information matrix (inverse of $E\left[\tilde{\tilde{\varepsilon}} \tilde{\boldsymbol{\varepsilon}}^{T}\right]$ ).

Recalling that the measured magnetic vector $\boldsymbol{r}_{i}^{B}$ does not change much in a short period, we can deduce two conclusions from Eq. (15).

- The attitude estimation error perpendicular to $\boldsymbol{r}_{i}$ is approximately evaluated as $\sigma_{v} / \sqrt{N}$

- The attitude estimation error parallel to $\boldsymbol{r}_{i}$ depends on the amount of changes in $\boldsymbol{r}_{i}$ and can be fairly large.

Hereafter the superscript ${ }^{B}$ is intentionally deleted, because the attitude estimation error is factorized into two components such that one is perpendicular to $\boldsymbol{r}_{i}^{B}$ and the other is parallel to $\boldsymbol{r}_{i}^{B}$, whose expression does not actually depend on the coordinates used for $\boldsymbol{r}_{i}$.

In order to evaluate quantitatively how large the attitude estimation error parallel to $\boldsymbol{r}_{i}$ is, we approximate the changes in $\boldsymbol{r}_{i}$ as a successive small angle rotation around a unit vector $\lambda$, which is defined as

$$
\lambda=\frac{\boldsymbol{r} \times(d \boldsymbol{r} / d t)}{\|\boldsymbol{r} \times(d \boldsymbol{r} / d t)\|}
$$

where $\boldsymbol{r}$ is a representative (e.g. averaged values for $\boldsymbol{r}_{i}$ ) magnetic vector and $(d \boldsymbol{r} / d t)$ means a direction change of $\boldsymbol{r}$ in a very short time. Therefore, $(d \boldsymbol{r} / d t)$ is perpendicular to $\boldsymbol{r}$. The magnetic vector at i-th sampling $\boldsymbol{r}_{i}$ is described as

$$
\boldsymbol{r}_{i}=\left(\cos \theta_{i} \boldsymbol{I}_{3}+\sin \theta_{i}[\lambda \times]\right) \boldsymbol{r} .
$$

Here we assume that each successive small angle rotation $\delta \theta_{i}$ is identical and given by

$$
\delta \theta_{i}=\frac{\theta}{N-1} .
$$

Then, $\theta_{i}$ is described as

$$
\theta_{i}=\left(i-\frac{N+1}{2}\right) \delta \theta, \quad i=1, \cdots, N .
$$

Substituting Eq. (17) into Eq. (15) and considering that $N$ is a large number, we obtain explicit expressions for statistical evaluation of attitude estimation errors as

$$
E\left[\tilde{\boldsymbol{\varepsilon}} \tilde{\boldsymbol{\varepsilon}}^{T}\right]=\frac{\sigma_{v}^{2}}{N}\left(\lambda \lambda^{T}+\frac{12}{\theta^{2}} \boldsymbol{r} \boldsymbol{r}^{T}+\frac{1}{1-\theta^{2} / 12}(d \boldsymbol{\boldsymbol { r }} / d t)(d \boldsymbol{r} / d t)^{T}\right) .
$$

From Eq. (20), it is concluded that

- On the right side of Eq. (20), the common factor, $\sigma_{v}^{2} / N$ shows statistical improvement of estimation accuracy when the number of measurements increases, whereas terms in the parenthesis shows the breakdown of the estimation error among the three components, parallel to $\lambda$, parallel to $\boldsymbol{r}$, and parallel to $d \overline{\boldsymbol{r}} / d t$.

- The attitude estimation error parallel to $\lambda$ is the smallest component and is evaluated as $\sigma_{v} / \sqrt{N}$.

- The attitude estimation error parallel to $\boldsymbol{r}$ is the largest component and is evaluated as $2 \sqrt{3} \sigma_{v} /(\sqrt{N} \theta)$. The error is in inverse proportion to the rotational angle (the amount of direction change) of the magnetic vector. This justifies the intuitive requirement that to estimate a three-axis attitude it is necessary to wait for a while so that the orbital motion of the spacecraft brings a well different position in the earth magnetic field. The factor $2 \sqrt{3} / \theta$ depends on altitude, inclination angle, position in the orbit and total 
measurement period. For example, at altitude $900 \mathrm{~km}$ and with total measurement period $600 \mathrm{~s}, 2 \sqrt{3} / \theta$ is about 5 to 15 for inclination angle 10 degrees and about 2 to 4 for inclination angle 90 degrees, depending on the position in the orbit.

- The attitude estimation error parallel to $d \overline{\boldsymbol{r}} / d t$ is evaluated as $\sigma_{v} / \sqrt{N\left(1-\theta^{2} / 12\right)}$. Because $\theta$ is much smaller than 1 , this error is just a little larger than the error parallel to $\lambda$.

\section{Rotating Attitude Determination by Magnetic Sensors and Rate Sensors}

We extend the attitude determination algorithm developed in section 2 to combine magnetic sensors with rate sensors in order to determine a three-axis attitude of spacecraft whose attitude can not be regarded as stationary. The basic idea is to use rate sensor outputs in order to compensate for the rotational motion so that the problem of attitude determination is reduced similar to a stationary one (Fig. 3).

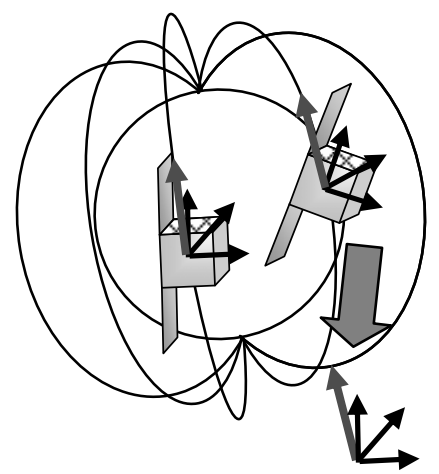

Fig. 3. Measurement at two points (rotating case)

\subsection{Measurement model}

Rate sensors measure the body rates of the spacecraft in the body coordinates. Their outputs contain a bias, which can be regarded as constant during a short time period

$$
\boldsymbol{\omega}_{i}^{\text {mes }}=\boldsymbol{\omega}_{i}+\boldsymbol{\omega}_{b}
$$

In combination with the measured body rates $\boldsymbol{\omega}_{i}^{\text {mes }}$, the relationships between the measured magnetic field vector and the reference vector become

$$
\begin{gathered}
\boldsymbol{r}_{i}^{B}=\boldsymbol{U}_{I \rightarrow B\left(t_{i}\right)} \boldsymbol{r}_{i}^{I}=\boldsymbol{U}_{B\left(t_{1}\right) \rightarrow B\left(t_{i}\right)} \boldsymbol{U}_{I \rightarrow B\left(t_{1}\right)} \boldsymbol{r}_{i}^{I} \\
\boldsymbol{U}_{B\left(t_{1}\right) \rightarrow B\left(t_{i}\right)}=\boldsymbol{U}\left(\boldsymbol{\omega}_{i-1}^{\text {mes }}-\boldsymbol{\omega}^{b} \Delta t\right) \times \cdots \\
\quad \times \boldsymbol{U}\left(\boldsymbol{\omega}_{2}^{\text {mes }}-\boldsymbol{\omega}^{b} \Delta t\right) \times \boldsymbol{U}\left(\boldsymbol{\omega}_{1}^{\text {mes }}-\boldsymbol{\omega}^{b} \Delta t\right) .
\end{gathered}
$$

When a rate sensor is allocated to each axis, the unknown variables are the three-axis attitude and three biases, which means 6 unknowns in total. Therefore, it is necessary to take measurements at least three points that are enough separated from each other. To average out the measurement errors and model errors contained in Eqs. (21) and (22), we take $N$ measurements. Then the attitude estimation problem is to find an initial coordinates transformation matrix $\boldsymbol{U}_{B\left(t_{1}\right) \rightarrow I}$ and a bias vector $\boldsymbol{\omega}_{b}$ that satisfy Eqs. (21) and (22) for $i=1 \cdots N$.
Then we can compute a coordinates transformation matrix at i-th sampling time as

$$
\boldsymbol{U}_{I \rightarrow B\left(t_{i}\right)}=\boldsymbol{U}_{B\left(t_{1}\right) \rightarrow B\left(t_{i}\right)} \boldsymbol{U}_{I \rightarrow B\left(t_{1}\right)} .
$$

\subsection{Attitude determination algorithm}

Similar to subsection 2.2 , we define a cost function $J$ and find $\boldsymbol{U}_{B\left(t_{1}\right) \rightarrow I}$ and $\boldsymbol{\omega}_{b}$ that minimize $J$.

$$
\min _{\boldsymbol{U}_{B(1) \rightarrow l}, \boldsymbol{\omega}_{b}} J\left(\boldsymbol{U}_{B\left(t_{1}\right) \rightarrow I}, \boldsymbol{\omega}_{b}\right)=\sum_{i=1}^{N}\left\|\boldsymbol{r}_{i}^{I}-\boldsymbol{U}_{B\left(t_{i}\right) \rightarrow I} \boldsymbol{r}_{i}^{B}\right\|^{2}
$$

We first assume $\boldsymbol{\omega}_{b}$ is zero and compensate the rotational motion of the spacecraft by applying the following coordinates transformation.

$$
\boldsymbol{r}_{2}^{B} \rightarrow \boldsymbol{U}_{B\left(t_{1}\right) \rightarrow B\left(t_{2}\right)}^{T} \boldsymbol{r}_{2}^{B}
$$

With this rotation-compensated vector, we compute the initial estimate of $\hat{\boldsymbol{U}}_{B\left(t_{1}\right) \rightarrow I}$ using algebraic method.

Then we update the attitude estimates in the following way.

$$
\begin{gathered}
\boldsymbol{U}_{B\left(t_{1}\right) \rightarrow I}=\hat{\boldsymbol{U}}_{B\left(t_{1}\right) \rightarrow I} \delta \boldsymbol{U} \\
\boldsymbol{\omega}_{i}=\boldsymbol{\omega}_{i}^{\text {mes }}-\boldsymbol{\omega}_{b}-\delta \boldsymbol{\omega}_{b} \\
\delta \boldsymbol{U} \approx \boldsymbol{I}_{3}-[\boldsymbol{\varepsilon} \times]
\end{gathered}
$$

Similar to section 2, we derive linearized equations in terms of attitude estimate update and a bias vector.

$$
\min _{U_{B\left(t_{1}\right) \rightarrow l}, \omega_{b}} J\left(\varepsilon, \delta \omega_{b}\right)=\sum_{i=1}^{N}\left\|\Delta r_{i}^{B}-B_{i} \varepsilon-C_{i} \delta \omega_{b}\right\|^{2}
$$

where a residual vector $\Delta \boldsymbol{r}_{i}^{B}$ and matrices $\boldsymbol{B}_{i}$ and $\boldsymbol{C}_{i}$ are defined as

$$
\begin{aligned}
\Delta \boldsymbol{r}_{i}^{B} & =\hat{\boldsymbol{U}}_{B\left(t_{1}\right) \rightarrow I}^{T} \boldsymbol{r}_{i}^{I}-\boldsymbol{U}_{B\left(t_{1}\right) \rightarrow B\left(t_{i}\right)}{ }^{T} \boldsymbol{r}_{i}^{B} \\
\boldsymbol{B}_{i} & =\left[\left(\boldsymbol{U}_{B\left(t_{1}\right) \rightarrow B\left(t_{i}\right)}{ }^{T} \boldsymbol{r}_{i}^{B}\right) \times\right] \\
\boldsymbol{C}_{i} & =\left[\left(\boldsymbol{U}_{B\left(t_{1}\right) \rightarrow B\left(t_{i}\right)}{ }^{T} \boldsymbol{r}_{i}^{B}\right) \times\right] \Delta t \\
& +\boldsymbol{U}_{B\left(t_{1}\right) \rightarrow B\left(t_{2}\right)}{ }^{T}\left[\left(\boldsymbol{U}_{B\left(t_{2}\right) \rightarrow B\left(t_{i}\right)}{ }^{T} \boldsymbol{r}_{i}^{B}\right) \times\right] \Delta t \\
& +\cdots+\boldsymbol{U}_{B\left(t_{1}\right) \rightarrow B\left(t_{i-1}\right)}{ }^{T}\left[\left(\boldsymbol{U}_{B\left(t_{i-1}\right) \rightarrow B\left(t_{i}\right)}{ }^{T} \boldsymbol{r}_{i}^{B}\right) \times\right] \Delta t .
\end{aligned}
$$

Following the standard least square method, we obtain the optimal attitude update $\hat{\boldsymbol{\varepsilon}}$ and a bias update $\delta \boldsymbol{\omega}_{b}$ as

$$
\begin{aligned}
& {\left[\begin{array}{c}
\hat{\boldsymbol{\varepsilon}} \\
\boldsymbol{\delta} \boldsymbol{\omega}_{b}
\end{array}\right]=\left[\begin{array}{ll}
\boldsymbol{D}_{0} & \boldsymbol{D}_{1} \\
\boldsymbol{D}_{1} & \boldsymbol{D}_{2}
\end{array}\right]^{-1}\left[\begin{array}{c}
\boldsymbol{d}_{0} \\
\boldsymbol{d}_{1}
\end{array}\right]} \\
& \boldsymbol{D}_{0}=\sum_{i=1}^{N} \boldsymbol{B}_{i}^{T} \boldsymbol{B}_{i}, \quad \boldsymbol{d}_{0}=\sum_{i=1}^{N} \boldsymbol{B}_{i}^{T} \Delta \boldsymbol{r}_{i}^{B} \\
& \boldsymbol{D}_{1}=\sum_{i=1}^{N} \boldsymbol{C}_{i}^{T} \boldsymbol{B}_{i}, \quad \boldsymbol{d}_{1}=\sum_{i=1}^{N} \boldsymbol{C}_{i}^{T} \Delta \boldsymbol{r}_{i}^{B} \\
& \boldsymbol{D}_{2}=\sum_{i=1}^{N} \boldsymbol{C}_{i}^{T} \boldsymbol{C}_{i} .
\end{aligned}
$$

We can use a new estimate computed from Eqs. (27) and (28) and iterate the process of Eqs. (31)-(35) to improve estimation accuracy. 


\subsection{Evaluation of estimation accuracy}

Similarly to subsection 2.3 , statistical evaluation of attitude and bias estimation errors is obtained as

$$
\begin{gathered}
E\left[\begin{array}{c}
\hat{\boldsymbol{\varepsilon}} \\
\delta \boldsymbol{\omega}_{b}
\end{array}\right]=\left[\begin{array}{l}
\boldsymbol{0} \\
\boldsymbol{0}
\end{array}\right] \\
E\left[\left(\begin{array}{c}
\tilde{\boldsymbol{\varepsilon}} \\
\delta \tilde{\boldsymbol{\omega}}_{b}
\end{array}\right)\left(\begin{array}{c}
\tilde{\boldsymbol{\varepsilon}} \\
\delta \tilde{\boldsymbol{\omega}}_{b}
\end{array}\right)^{T}\right]=\sigma_{v}^{2}\left[\begin{array}{cc}
\boldsymbol{D}_{0} & \boldsymbol{D}_{1} \\
\boldsymbol{D}_{1} & \boldsymbol{D}_{2}
\end{array}\right]^{-1}
\end{gathered}
$$

This expression is an extended one from Eq. (13). Its validity is verified in the numerical examples given in section 4.

\section{Numerical Examples}

\subsection{Simulation conditions}

To evaluate the proposed algorithm and statistical analysis of estimation accuracy, Monte-Carlo simulations are conducted.

The simulation conditions for stationary spacecraft and rotating spacecraft are summarized in Tables 1 and 2 . In the tables, underlined items are chosen for parametric study and underlined values are the default ones. Initial attitude (quaternion) and rate sensor bias are randomly chosen. Dipole model is used as a geomagnetic field model.

\subsection{Simulation results for stationary spacecraft}

Here we apply the estimation algorithm described in section 2 , where the spacecraft is assumed stationary.

Figures 4, 5, and 6 are the results of parametric study and they show attitude estimation error with respect to magnetic sensor output error, total measurement time, and number of measurements for a stationary spacecraft. In the figures, the estimation errors are divided into three components, i.e. parallel to $\boldsymbol{r}$, parallel to $d \overline{\boldsymbol{r}} / d t$, and parallel to $\lambda$. A dot represents an each sample from simulations. Short lines show $\pm 1 \sigma$ values computed from Eq. (20). From the figures, it is shown that

- Attitude estimation error is in proportion to magnetic sensor output error.

- Estimation error parallel to $\boldsymbol{r}$ is in inverse proportion to the total measurement time.

- Estimation error perpendicular to $\boldsymbol{r}$ is not affected by the total measurement time.

- Because the total change in the magnetic vector direction is approximately in proportion to the total simulation time, the two findings above are both explained by the discussions about Eq. (20).

- All estimation errors are in inverse proportion to the squared root of number of measurements. This is explained by the discussions about Eq. (20).

- Sigma values match the simulation results.

\subsection{Simulation results for rotating spacecraft}

Here we apply the estimation algorithm in section 3, where both magnetic sensors and rate sensors are used.

Figure 7 is the result of parametric study. It shows attitude estimation error and body rate estimation error (or the rate sensor bias estimation error), with respect to magnetic sensor output error for a rotating spacecraft. The ordinates and symbols in the figure are similar to Fig. 4 except that the sigma values are computed from Eq. (37). From the figures, it is shown that

- Both attitude and body rate estimation errors are in proportion to magnetic sensor output error.

- Body rate estimation errors are on the same order for each axis.

- Attitude estimation error increases when rate bias is estimated, considering that the sampling period is shortened from 10 to 2 seconds.

\begin{tabular}{|c|c|c|}
\hline item & unit & Value \\
\hline orbit height & $\mathrm{km}$ & 1000 \\
\hline eccentricity & & 0 \\
\hline inclination & degree & 30 \\
\hline $\begin{array}{l}\text { magnetic sensor } \\
\text { output error }\end{array}$ & $\begin{array}{l}\text { rad-sigma } \\
\text { per axis }\end{array}$ & $0.01,0.025, \underline{0.05}, 0.10$ \\
\hline initial attitude & & $(-0.247,-0.952,0.072,0.164)$ \\
\hline initial body rate & degree/s & $(0,0,0)$ \\
\hline $\begin{array}{l}\text { total measurement } \\
\text { period }\end{array}$ & $\mathrm{s}$ & $120,300, \underline{600}, 1200$ \\
\hline sampling period & $\mathrm{s}$ & $2,5, \underline{10}, 20$ \\
\hline
\end{tabular}

- Sigma values match the simulation results.

Table 2. Simulation condition for rotating spacecraft.

\begin{tabular}{|l|l|l|}
\hline item & unit & Value \\
\hline orbit height & $\mathrm{km}$ & 1000 \\
\hline eccentricity & & 0 \\
\hline inclination & degree & 30 \\
\hline $\begin{array}{l}\text { magnetic sensor } \\
\text { output error }\end{array}$ & $\begin{array}{l}\text { rad-sigma } \\
\text { per axis }\end{array}$ & $0.01,0.025, \underline{0.05}, 0.10$ \\
\hline rate sensor bias & degree/s & $(0.1,0.1,0.1)$ \\
\hline initial attitude & degree/s & $(2,2,2)$ \\
\hline initial body rate & $\mathrm{s}$ & 600 \\
\hline $\begin{array}{l}\text { total measurement } \\
\text { period }\end{array}$ & $\mathrm{s}$ & 2 \\
\hline sampling period & &
\end{tabular}

\section{Conclusion}

We proposed a new attitude determination algorithm for a three-axis spacecraft in low earth orbits using magnetic sensors and rate sensors. First, an attitude determination algorithm using only magnetic sensors was introduced for a stationary attitude. Then the algorithm was extended to combine magnetic sensors with rate sensors, where both the three-axis attitude and rate sensor biases are estimated. The estimation error was statistically analyzed. Numerical examples showed validity of the attitude determination algorithm and its accuracy evaluation. 


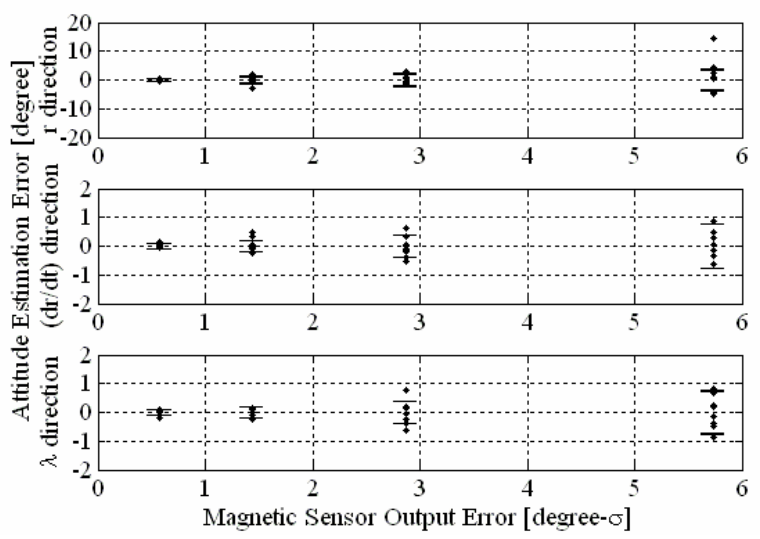

Fig. 4. Attitude estimation error with respect to magnetic sensor output error (stationary case)

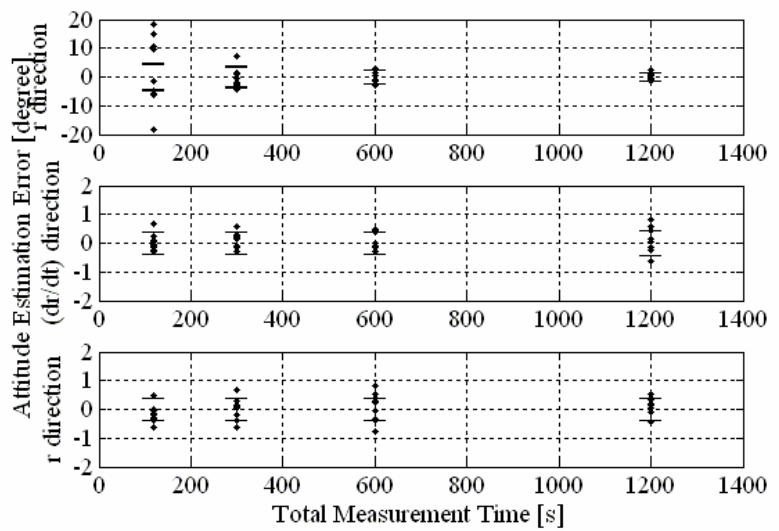

Fig. 5. Attitude estimation error with respect to total measurement time (stationary case)

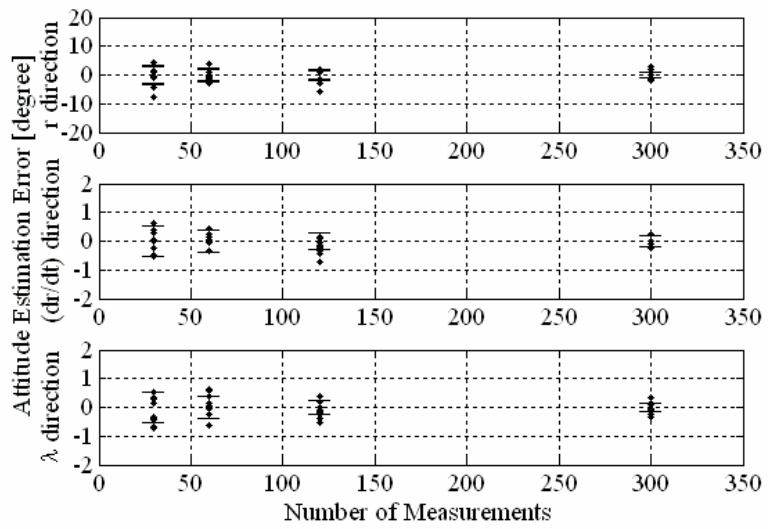

Fig. 6. Attitude estimation error with respect to number of measurements (stationary case)

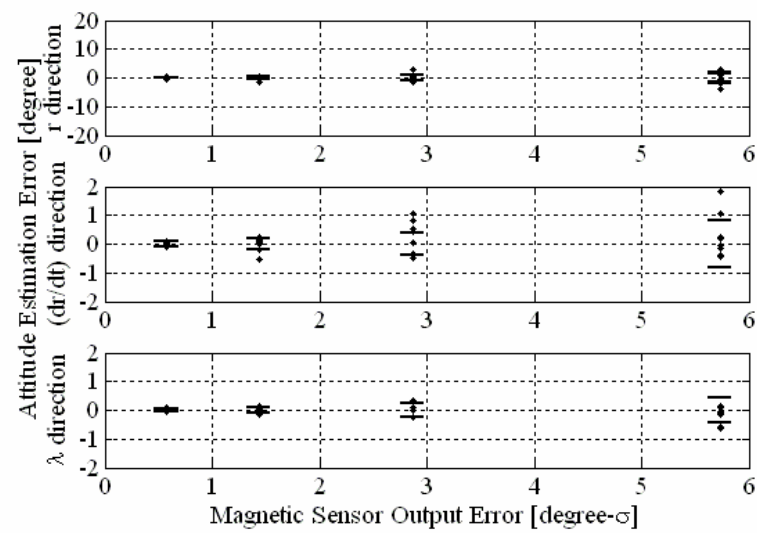

a) Attitude estimation error

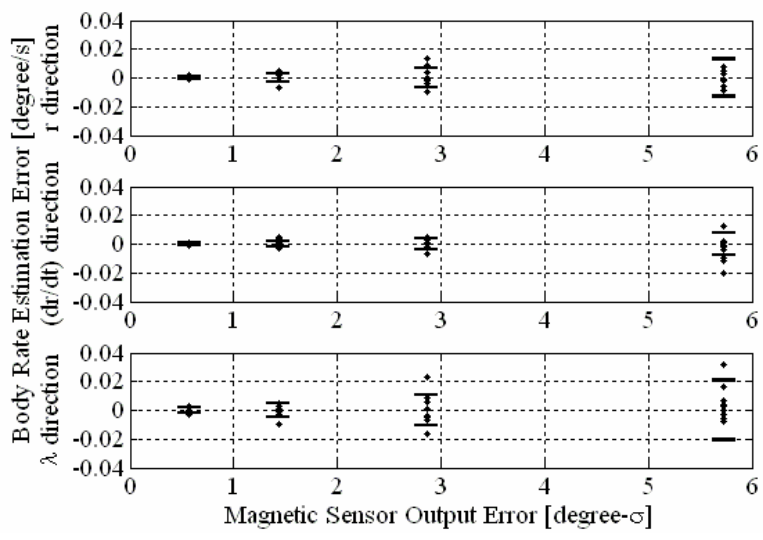

b) Body rate estimation error

Fig. 7. Attitude and body rate estimation error (rotating case)

\section{References}

1) Merayo, J., Brauer, P., Joergensen, P., Risbo, T. and Cain, J.: The Spinning Astrid-2 Satellite Used for Modeling the Earth's Main Magnetic Field, IEEE Trans. Geoscience and Remote Sensing, 40 (2002), pp. 898-909.

2) Challa, M., Kotaru, S., and Natanson, G.: Magnetometer-Only Attitude and Rate Estimates during the Earth Radiation Budget Satellite 1987 Control Anomaly, AIAA Paper 97-3615, 1997.

3) Bar-Itzhack, I. and Harman, R.: Recursive Attitude and Rate Determination of Spinning Spacecraft, AIAA Guidance, Navigation and Control Conference and Exhibit, AIAA Paper 2007-6811, AIAA, Reston, VA, 2007.

4) Oshman, Y. and Dellus, F.: Fast Estimation of Spacecraft Angular Velocity from Sequential Geomagnetic Field Observations, AIAA Paper 2000-4242, AIAA, Reston, VA, 2000.

5) Challa, M., Natanson, G. and Ottenstein, N.: Magnetometer-Only Attitude and Rate Estimates for Spinning Spacecraft, AIAA Paper 2000-4241, AIAA, Reston, VA, 2000.

6) Wertz, J. (ed.): Spacecraft Attitude Determination and Control, pp. 118-119, 249-254, 424-426, D. Reidel Pub. Co., Boston, 1978.

7) Challa, M. and Harman, R.: A New Magnetometer Calibration Algorithm and Applications, Proc. of AIAA Guidance, Navigation, and Control Conference, Boston, MA, 1998, pp. 697-707.

8) http://www.ngdc.noaa.gov/IAGA/vmod/igrfhw.html

9) Shuster, M.: An Efficient Algorithm for Spacecraft Attitude Determination with Optical Sensors, Advances in the Astronautical Sciences, 1998, pp. 407-419. 